\title{
Enrichment and characterization of a bacterial culture that can degrade 4-aminopyridine
}

\author{
Shinji Takenaka*, Ryosuke Nomura, Ayumi Minegishi and Ken-ichi Yoshida
}

\begin{abstract}
Background: The agrichemical 4-aminopyridine is used as a bird repellent in crop fields and has an epileptogenic action in a variety of animals, including man and mouse. 4-Aminopyridine is biodegraded in the environment through an unknown mechanism.

Results: A 4-aminopyridine-degrading enrichment culture utilized 4-aminopyridine as a carbon, nitrogen, and energy source, generating 4-amino-3-hydroxypyridine, 3,4-dihydroxypyridine, and formate as intermediates. 4-Amino-3-hydroxypyridine could not be further metabolized and probably accumulated as a dead-end product in the culture. Biodegradability tests and partial sequence analysis of the enrichment culture indicated that 4-aminopyridine was mainly degraded via 3,4-dihydroxypyridine and that the metabolite is probably cleaved by 3-hydroxy-4-pyridone dioxygenase. Seven culturable predominant bacterial strains (strains 4AP-A to 4AP-G) were isolated on nutrient agar plates. Changes in the bacterial populations of 4-aminopyridine, 3,4-dihydroxypyridine, or formate/ammonium chloride enrichment cultures were monitored by denaturing gradient gel electrophoresis (DGGE) profiling of PCR-amplified $16 \mathrm{~S}$ rRNA gene fragments. Sequence analysis of the $16 \mathrm{~S}$ rRNA gene fragments derived from predominant DGGE bands indicated that Pseudomonas nitroreducens 4AP-A and Enterobacter sp. 4AP-G were predominant in the three tested enrichment cultures and that the unculturable strains Hyphomicrobium sp. 4AP-Y and Elizabethkingia sp. 4AP-Z were predominant in 4-aminopyridine and formate/ammonium chloride enrichment cultures and in the 3,4-dihydroxypyridine enrichment culture, respectively. Among the culturable strains, strain 4AP-A could utilize 3,4-dihydroxypyridine as a growth substrate. Although we could not isolate strain 4AP-Y on several media, PCR-DGGE analysis and microscopy indicated that the unique bi-polar filamentous bacterial cells gradually became more dominant with increasing 4-aminopyridine concentration in the medium.
\end{abstract}

Conclusions: Hyphomicrobium sp. 4AP-Y, P. nitroreducens 4AP-A, and Elizabethkingia sp. 4AP-Z probably play important roles in 4-aminopyridine degradation in crop fields. In the enrichment culture, 3,4-dihydroxypyridine and its metabolites including formate might be shared as growth substrates and maintain the enrichment culture, including these indispensable strains.

Keywords: 4-aminopyridine, 4-amino-3-hydroxypyridine, 3,4-dihydroxypyridine, Hyphomicrobium, Pseudomonas nitroreducens, Elizabethkingia

\section{Background}

Pyridine and its derivatives are mainly produced on an industrial scale from coal tar. These compounds are major industrial raw materials and intermediates used for organic solvents and the production of agrichemicals, medicines, and active surfactants [1]. Pyridines are soluble in polar and nonpolar solvents, and most are toxic [2].

\footnotetext{
* Correspondence: hakko3@kobe-u.ac.jp

Department of Applied Biological Chemistry, Graduate School of Agricultural Science, Kobe University, 1-1 Rokkodai, Nada-ku, Kobe 657-8501, Japan
}

Pyridine and its derivatives are also environmental pollutants, and their biodegradation has been studied in detail [3]. The biodegradability of pyridine derivatives follows the order pyridinecarboxylic acids $>$ pyridine $=$ monohydroxypyridines > methylpyridines > aminopyridines $=$ chloropyridines [4]. Generally, pyridines are degraded via pyridine-ring reduction and fission steps [5] or via pyridine-ring hydroxylation and fission steps [6-8]. Nocardia sp. strain Z1 directly cleaves the pyridine ring between $\mathrm{N}$ and position $\mathrm{C}-2$ and further metabolizes the product via glutaric dialdehyde, and Bacillus sp.

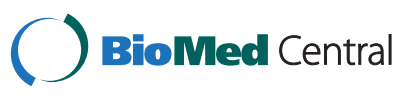


strain 4 cleaves the ring between positions $\mathrm{C}-2$ and $\mathrm{C}-3$ and the product it further via succinate semialdehyde [9]. Gordonia nitida strain LE31 metabolizes 3-methylor 3-ethyl-pyridine without a hydroxylation step [5]. Rhodococcus opacus (VKM Ac-1333D) and Arthrobacter crystallopoietes (VKM Ac-1334D) hydroxylate the pyridine ring [8]. In Agrobacterium sp. strain NCIB 10413, 4-hydroxypyridine is metabolized by a hydroxylase and an $N$-heterocyclic ring-cleavage dioxygenase [6,7]. Thus, the biodegradation of pyridines by single bacterial species has been studied, but little is known about the biodegradation of pyridines by microbial communities [10], which could include unculturable bacteria.

Aminopyridines are persistent chemical [4] and are a class of potentially genotoxic impurities in pharmaceutical products [11]. 4-Aminopyridine (Figure 1, compound I) has been marketed for agricultural use as Avitrol and used for repelling and killing bird pests [12]. The compound is a potassium-channel blocker [13] and has epileptogenic action in a variety of animals, including man and mouse $[14,15]$. However, the metabolic fate of 4-aminopyridine in an ecosystem [16] and its biodegradation by an isolated a bacterium or bacterial community has not been studied in detail. It is broken down slowly by soil microorganisms in 2 months [16]. Here we report the enrichment and adaptation of a 4-aminopyridine-degrading enrichment culture and the characterization of the bacterial populations under different culture conditions.

\section{Methods}

\section{Organisms and growth conditions}

Enrichments of 4-aminopyridine-degrading bacteria were set up with $0.2 \mathrm{~g}$ normal farm soils such as rice field soil and corn field soils from the Hyogo Prefecture, Japan in $7 \mathrm{ml}$ basal medium containing $2.13 \mathrm{mM}$ (0.02\% wt/vol) 4-aminopyridine as described previously [17]. Briefly, solutions A (sodium-potassium phosphate solution), B (metal-salt solution containing $1 \mathrm{ml}$ of a soil extract), and C (4-aminopyridine solution) were prepared separately. The soil extract used in solution B was prepared by adding $15 \mathrm{~g}$ of a normal rice field soil to $200 \mathrm{ml}$ of deionized water and mixing for $30 \mathrm{~min}$, followed by filtration through Whatman No. 2 filter paper (Maidstone, UK) and autoclaving.

Ten 4-aminopyridine-degrading enrichment cultures, KM20-14A to KM20-14J, were incubated at $30^{\circ} \mathrm{C}$ with shaking at $140 \mathrm{rpm}$. Every 4 days, $500 \mu \mathrm{l}$ of the enrichment culture was used to inoculate $7 \mathrm{ml}$ fresh medium, to maintain 4-aminopyridine degradation ability. We selected one enrichment culture derived from a normal rice field soil, No. KM 20-14E for further study and examined its utilization of the identified metabolites (4-amino-3hydroxypyridine and 3,4-hydroxypyridine) by the enrichment culture (No. KM20-14E) was examined. The tested substrate was added to the basal medium instead of 4-aminopyridine.

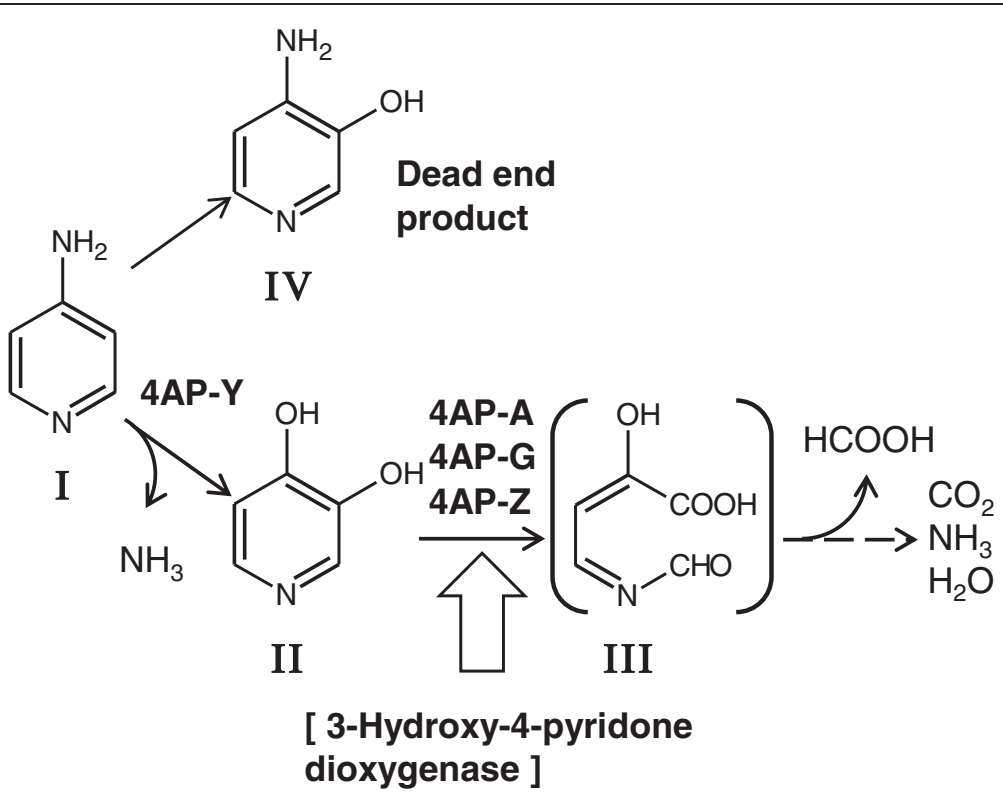

Figure 1 Proposed pathway of 4-aminopyridine degradation by the enrichment culture. I, 4-aminopyridine; II, 3,4-dihydroxypyridine; III, 3-(N-formyl)-formiminopyruvate; and IV, 4-amino-3-hydroxypyridine. The ring-cleavage product 3-(N-formyl)-formiminopyruvate from 3, 4-dihydroxypyridine was hypothesized from the metabolic pathway of 3,4-dihydroxypyridine in Agrobacterium sp. NCIB 10413 [6,7]. The strains of the enrichment culture probably involved in the steps are indicated. 
Isolation and identification of culturable and unculturable strains from the 4-aminopyridine-degrading enrichment culture

Samples taken from the 4-aminopyridine-degrading enrichment culture were serially diluted $10^{6}$ - to $10^{8}$-fold with $0.8 \%$ (wt/vol) $\mathrm{NaCl}$ solution and spread onto nutrient agar plates (1.0 g polypeptone, $1.0 \mathrm{~g}$ meat extract, $0.5 \mathrm{~g} \mathrm{NaCl}$, and $1.5 \mathrm{~g}$ agar per $100 \mathrm{ml}$ ), $0.1 \%$ (wt/vol) 4-aminopyridine agar plates, and $0.1 \%$ (wt/vol) 3,4-dihydroxypyridine agar plates. The plates were incubated at $30^{\circ} \mathrm{C}$ for 4 to 7 days, and colonies were picked up for $16 \mathrm{~S}$ rRNA gene analysis. We designated seven dominant bacterial strains isolated from the nutrient agar plate as dominant bacterial strains 4AP-A to 4AP-G. The $16 \mathrm{~S}$ rRNA gene V3 regions derived from these strains were used as a PCR-DGGE analysis makers as described below.

The isolates were characterized by physiological and biochemical parameters, such as gram reaction, flagella type, catalase activity, oxidase activity, OF test, fluorescent pigment production, and hydrolysis of gelatin, starch, and urea, following classical methods and by $16 \mathrm{~S}$ rRNA gene analysis [18] (see Additional file 1: Tables S1 and S2). Minor or unculturable strains were classified only by $16 \mathrm{~S}$ rRNA gene analysis. $16 \mathrm{~S}$ rRNA genes were amplified using the universal primers $\mathrm{pA}$ and $\mathrm{pH}^{\prime}$ [18] (Table 1), and their nucleotide sequences (approximately $1,500 \mathrm{bp}$ ) were determined and compared to sequences in the DDBJ/EMBL/GenBank database.

\section{Isolation, and identification of metabolites from 4-aminopyridine}

The enrichment culture was cultivated in basal medium containing $2.13 \mathrm{mM}$ 4-aminopyridine at $30^{\circ} \mathrm{C}$ with shaking, and the culture was diluted $10^{6}$ to $10^{8}$-fold with $0.8 \%$ (wt/vol) $\mathrm{NaCl}$ solution. The diluted culture $(500 \mu \mathrm{l})$ was used to inoculate fresh basal medium containing 4aminopyridine, and the subculture was incubated at $30^{\circ} \mathrm{C}$. The culture was centrifuged at $20,000 \times g$ for 10 min, and the supernatant was dried using a rotary evaporator. The dried residues were dissolved in $n$-butanol and then dried again. The accumulated products in the dried residue were incubated with $\mathrm{N}, \mathrm{O}$-bis(trimethylsilyl) trifluoroacetamide at $100^{\circ} \mathrm{C}$ for $1.5 \mathrm{~h}$. The trimethylsilylated products were analyzed by GC-MS as described below.

\section{Measurement and identification of 4-aminopyridine and its metabolites}

Concentrations of pyridines, including 4-aminopyridine and 4-amino-3-hydroxypyridine (Figure 1, compound IV), were measured using a Hitachi L-6200 HPLC system (Tokyo, Japan) equipped with a Cosmosil 5C18 PAQ column $(4.6 \times 150 \mathrm{~mm}$; Nacalai Tesque, Kyoto). The eluent was $20 \mathrm{mM}$ potassium phosphate buffer $(\mathrm{pH} 2.5)$
Table 1 Oligonucleotide primers used in this study

\begin{tabular}{|c|c|c|}
\hline Primer & Sequence ( $5^{\prime}$ to $\left.3^{\prime}\right)$ & Reference \\
\hline \multirow[t]{2}{*}{$\mathrm{pA}$} & AGAGTTTGATCCTGGCTCAG & [7] \\
\hline & $(8-28)$ & \\
\hline \multirow[t]{2}{*}{$\mathrm{pH}^{\prime}$} & AAGGAGGTGATCCAGCCGCA & [7] \\
\hline & $(1542-1522)$ & \\
\hline PRBA338GCf & $\begin{array}{l}\text { CGCCCGCCGCGCGCGGCGGGCGGGG } \\
\text { CGGGGGCACGGGGGGACTCCTACGG } \\
\text { GAGGCAGCAG }\end{array}$ & This study \\
\hline PRBA338f & TACGGGAGGCAGCAG & [26] \\
\hline PRUN518r & ATTACCGCGGCTGCTGG & {$[26]$} \\
\hline $\mathrm{PRSTY1}^{a}$ & ACGATAATGACGGTACCCGG & This study \\
\hline $\operatorname{PRSTY}^{a}$ & TTAGCCGGGACTTATTCTCC & This study \\
\hline $\mathrm{PRSTZ1}^{b}$ & TACTTACGTGTAAGTAGCTGAAGG & This study \\
\hline$\overline{\mathrm{PRSTZ2}^{b}}$ & CCTTCAGCTACTTACACGTAAGTA & This study \\
\hline PydAf ${ }^{c}$ & GAYGAYCAYTTYGARAAYCA & This study \\
\hline $\operatorname{PydAr}^{c}$ & CATICCRCADATCCAYTC & This study \\
\hline \multicolumn{3}{|c|}{$\begin{array}{l}\text { a Used for amplification of the full-length } 16 \mathrm{~S} \text { rRNA gene from strain 4AP-Y. } \\
{ }^{b} \text { Used for amplification of the full-length } 16 \mathrm{~S} \text { rRNA gene from strain 4AP-Z. } \\
{ }^{c} \text { PydAf and PydAr were designed based on the conserved regions of 3- } \\
\text { hydroxy-4-pyridone dioxygenase (3,4-dihydroxypyridine 2,3-dioxygenase), } \\
\text { DDHFENH and EWICGM, respectively. R is A or G; Y is C or T; D is A, G, or T; } \\
\text { and I is inosine. }\end{array}$} \\
\hline
\end{tabular}

containing $5 \mathrm{mM}$ pentanesulfonate; the flow rate was 1.0 $\mathrm{ml} / \mathrm{min}$. 4-Aminopyridine and 4-amino-3-hydroxypyridine were detected at $254 \mathrm{~nm}$ and had retention times of 5.4 and $7.6 \mathrm{~min}$, respectively. The metabolites from 4-aminopyridine (4-amino-3-hydroxypyridine and 3,4dihydroxypyridine; Figure 1) were identified and quantified using a GCMS-QP2010 Ultra (Shimadzu, Kyoto, Japan). A fused silica capillary column (InertCap 1MS; $0.25 \mathrm{~mm} \times 30 \mathrm{~m}$; GL Science) was used. Helium gas was the carrier at a linear velocity of $35 \mathrm{~cm} / \mathrm{s}$. The column temperature was programed from $50^{\circ} \mathrm{C}$ (held for $1 \mathrm{~min}$ ) to $280^{\circ} \mathrm{C}$ at a rate of $5^{\circ} \mathrm{C} / \mathrm{min}$ and then held at $280^{\circ} \mathrm{C}$ for $20 \mathrm{~min}$. The peaks derived from the trimethylsilylated derivatives of 4-aminopyridine, 4-amino-3-hydroxypyridine, and 3,4-dihydroxypyridine appeared at 18.2, 24.5, and 20.9 min, respectively. The organic acids in the culture supernatant were derivatized by pentafluorobenzyl bromide according to a previously reported method [19] and analyzed by GC-MS as described above. The peaks derived from the pentafluorobenzyl formate appeared at $8.5 \mathrm{~min}$.

\section{PCR-DGGE analysis}

(1)DNA extraction and PCR Aliquots $(1.5,1.0$, and $0.5 \mathrm{ml})$ of the enrichment culture were sampled at the early-, mid-, and lateexponential growth phases, respectively, and centrifuged. DNA in the cell pellets was extracted using Qiagen DNeasy Blood \& Tissue Kit according to the manufacturer's instructions (Nihon eido, 
Tokyo, Japan). The 16S rRNA genes were amplified from $0.5 \mu \mathrm{l}$ DNA by PCR ( $50 \mu$ reactions) using a Taq polymerase kit (TaKaRa BIO INC., Shiga, Japan) and the forward primer PRBA338GCf, which contains a GC clamp, and the reverse primer PRUN518r, which targets the V3 region of the 16S rRNA gene (Table 1); the primers were prepared as reported previously [20]. The following PCR protocol was used: initial denaturation at $95^{\circ} \mathrm{C}$ for 2 min; 35 cycles of denaturation at $95^{\circ} \mathrm{C}$ for $60 \mathrm{~s}$, annealing at $60^{\circ} \mathrm{C}$ for $30 \mathrm{~s}$, extension at $72^{\circ} \mathrm{C}$ for $30 \mathrm{~s}$; and final extension at $72^{\circ} \mathrm{C}$ for $5 \mathrm{~min}$. The $16 \mathrm{~S}$ rRNA genes of isolated strains were amplified by PCR of DNA isolated from colonies.

\section{(2)DGGE}

Approximately 100 to $200 \mathrm{ng}$ of each PCR product was analyzed by electrophoresis on 1.0-mm-thick polyacrylamide $[8 \%(\mathrm{wt} / \mathrm{vol})$ acrylamide-bisacrylamide (779:21)] DGGE gels following the manufacturer's protocol (Nihon Eido Co. Ltd., Tokyo, Japan). The denaturing gradient was from 27.5 to $42.5 \%$ [100\% corresponded to $7.08 \mathrm{M}$ urea and $40 \%$ (wt $/ \mathrm{vol}$ ) formamide]. Gels were subjected to a constant voltage of $50 \mathrm{~V}$ for $4 \mathrm{~h}$ at $60^{\circ} \mathrm{C}$. After electrophoresis, the gels were stained for $20 \mathrm{~min}$ in ethidium bromide solution. DNA was visualized under UV light, digitally captured, and analyzed using a Gel Imaging System (Nippon Genetics Co. Ltd., Tokyo, Japan).

(3)Cloning of PCR product and sequencing Prominent DNA bands from the DGGE gels were extracted and used as PCR templates with the forward primer PRBA338f without a GC clamp and the reverse primer PRUN518r. The nucleotide sequences obtained were compared with those of the 16S rRNA genes of the strains isolated. To analyze the full-length $16 \mathrm{~S}$ rRNA gene sequences, specific primers were designed based on the partial sequences of the isolate that became more dominant in the culture during continuous growth in basal medium containing 4-aminopyridine (Table 1).

\section{PCR amplification of part of the 3-hydroxy-4-pyridone dioxygenase gene}

The enrichment culture grown in 4-aminopyrdinecontaining medium was harvested in the mid-exponential growth phase by centrifugation. Mixed genomic DNA in the cell pellets was extracted using Qiagen DNeasy Blood \& Tissue Kit (Hilden, Germany) according to the manufacturer's instructions and was used as a template for PCR. To amplify part of the 3-hydroxy-4-pyridone dioxygenase (3,4-dihydroxypyridine 2,3-dioxygenase) gene, pydA, the primers PydAf and PydAr were designed based on the conserved region of previously reported dioxygenases from Rhizobium sp. TAL1145 (DDBJ/EMBL/GenBank accession no. AY729020), Hyphomicrobium sp. MC1 (YP_ 004673996), Bordetella bronchiseptica RB50 (NP_890665), and Bordetella parapertussis 12822 (NP_885852) (Table 1). The following PCR protocol was used: initial denaturation at $95^{\circ} \mathrm{C}$ for $2 \mathrm{~min} ; 35$ cycles of denaturation at $95^{\circ} \mathrm{C}$ for 60 $\mathrm{s}$, annealing at $45^{\circ} \mathrm{C}$ for $30 \mathrm{~s}$, extension at $72^{\circ} \mathrm{C}$ for $30 \mathrm{~s}$; and final extension at $72^{\circ} \mathrm{C}$ for $5 \mathrm{~min}$. Harvesting of cells, preparation of mixed genomic DNA, and amplification were carried out in triplicate.

\section{Analytical methods}

The optical density $\left(\mathrm{OD}_{660}\right)$ of the cultures was measured using a Hitachi U-2800 spectrophotometer. The ${ }^{1} \mathrm{H}-\mathrm{NMR}$ spectra of the isolated metabolites and the prepared standard compounds were measured with a Joel JNM-AL300 spectrometer (300 MHz, Joel Ltd., Tokyo, Japan). Released ammonia in the culture fluid was measured using the indophenol blue method [21]. Total protein in the culture was measured using the modified Lowry method, to confirm the utilization of 4aminopyridine as a carbon, nitrogen, and energy source by the enrichment culture [22].

\section{Nucleotide sequence accession numbers}

The nucleotide sequences of the 16S rRNA genes obtained in this study were deposited in the DDBJ/ EMBL/GenBank databases under accession numbers AB695349 through AB695357.

\section{Chemicals}

4-Aminopyridine and methyl chloroformate were purchased from Tokyo Chemical Industry (Tokyo, Japan). 4-Amino-3-hydroxypyridine hydrochloride was from SynChem OHG (Felsberg, Germany). L-Mimosine from Koa Hoale seeds and pentafluorobenzyl bromide were from Sigma Aldrich (St. Louis, MO, USA). 3,4Dihydroxypyridine was prepared from L-mimosine according to a previously reported method [23]. The ${ }^{1} \mathrm{H}$ NMR spectrum of the prepared 3,4-dihydroxypyridine was measured at NMR $\delta \mathrm{H}\left(\mathrm{DMSO}-d_{6}\right): \mathrm{dH}=7.35 \mathrm{ppm}$ (d, J = 6.0 Hz, 1H; H-6); 7.47 ppm (S, 1H; H-2); $6.21 \mathrm{ppm}$ (d, J = $6.0 \mathrm{~Hz} ; \mathrm{H}-5)$. N,O-bis(trimethylsilyl)trifluoroacetamide and pyridine derivatives were purchased from Wako Pure Chemicals (Osaka, Japan).

\section{Results}

\section{Degradation of 4-aminopyridine by the enrichment culture}

We selected one 4-aminopyridine-degrading enrichment culture from the ten enrichment cultures of soil samples incubated continuously with subculturing for 6 months. 
The enrichment culture grew well and could be maintained on basal medium containing 4-aminopyridine in the presence of soil extract. The culture degraded 4aminopyridine and used it as a carbon and nitrogen source (Figure 2).

\section{Identification and degradation of metabolites from 4-aminopyridine}

Two metabolites in the enrichment culture in medium containing 4-aminopyridine were detected using GC and GC-MS. The trimethylsilylated metabolites, compounds I and II, had GC retention times of 20.9 and $24.4 \mathrm{~min}$, respectively. Compound I was detected in the culture on the first day and accumulated during the cultivation. Compound II accumulated temporarily and was gradually degraded during cultivation. The mass spectrum of trimethylsilylated compound I showed a molecular ion at $m / z 254\left(\mathrm{M}^{+}\right.$, relative intensity $\left.81.3 \%\right)$. Major fragment ions appeared at $m / z 239\left(\mathrm{M}^{+}-\mathrm{CH}_{3}, 90 \%\right)$ and 73 $\left(\left[\mathrm{Si}\left(\mathrm{CH}_{3}\right)_{3}\right]^{+}, 100 \%\right)$. The mass spectrum of trimethylsilylated compound II showed a molecular ion at $\mathrm{m} / \mathrm{z}$ $255\left(\mathrm{M}^{+}\right.$, relative intensity $\left.25.7 \%\right)$. Major fragment ions appeared at $m / z 240\left(\mathrm{M}^{+}-\mathrm{CH}_{3}, 59.9 \%\right), 182\left(\mathrm{M}^{+}-\mathrm{Si}\left(\mathrm{CH}_{3}\right)_{3}\right.$, $1.1 \%), 147\left(\left[\left(\mathrm{CH}_{3}\right)_{2} \mathrm{Si}=\mathrm{O}-\mathrm{Si}\left(\mathrm{CH}_{3}\right)_{3}\right]^{+}, 2.1 \%\right)$, and $73([\mathrm{Si}$ $\left.\left.\left(\mathrm{CH}_{3}\right)_{3}\right]^{+}, 100 \%\right)$. The GC retention times and MS spectra of trimethylsilylated compounds I and II agreed with those of trimethylsilylated authentic 4-amino-3-hydroxypyridine and 3,4-dihydroxypyridine, respectively.

Pyridines are metabolized into an organic acid, such as acetate, formate, or dicarboxylic acids [4]. The culture supernatant of the enrichment culture was mixed with pentafluorobenzyl bromide and then analyzed. The mass spectrum of the pentafluorobenzyl derivative showed a molecular ion at $\mathrm{m} / z 226\left(\mathrm{M}^{+}\right)$. The GC retention time and MS spectrum of the derivatized compound agreed with those of formate derivatized by pentafluorobenzyl bromide. In the enrichment cultures grown on 2.12, 6.38, and $10.6 \mathrm{mM} 4$-aminopyridine for 10 days, $0.05 \pm$ $0.012 \mathrm{mM}$ formate accumulated in $10.6 \mathrm{mM}$ 4aminopyridine medium.

Although the enrichment culture gradually degraded 4aminopyridine with growth, 4-amino-3-hydroxypyridine accumulated in the culture to a final concentration of $6.4 \times 10^{-3} \mathrm{mM}$ after 5 days of cultivation. When we cultivated the enrichment culture in basal medium containing 4-amino-3-hydroxypyridine or 3,4-dihydroxypyridine (final concentration, $0.05 \% \mathrm{wt} / \mathrm{vol}$ ) with and without 4-aminopyridine, the culture completely degraded 3,4dihydroxypyridine in both media in 4 days but did not degrade 4-amino-3-hydroxypyridine in either medium.

\section{Identification of the gene encoding 3-hydroxy-4-pyridine dioxygenase in the isolated strains}

We hypothesized that 4-aminopyridine is metabolized to 3,4-dihydroxypyridine, and that the pyridine ring is then cleaved by 3-hydroxy-4-pyridone dioxygenase, as described below. The fragment amplified by pydA-specific primers was isolated and analyzed to determine whether some predominant strain in the enrichment culture carries the dioxygenase gene. The same sequence fragment was amplified from three different samples. The amino acid sequence deduced from the determined 801bp sequence showed a high level of identity with sequences of the extradiol-dioxygenase-3B-like superfamily of proteins, especially with that of the putative PydA from Hyphomicrobium sp. MC1 (YP_004673996) (see Additional file 2: Figure S1).

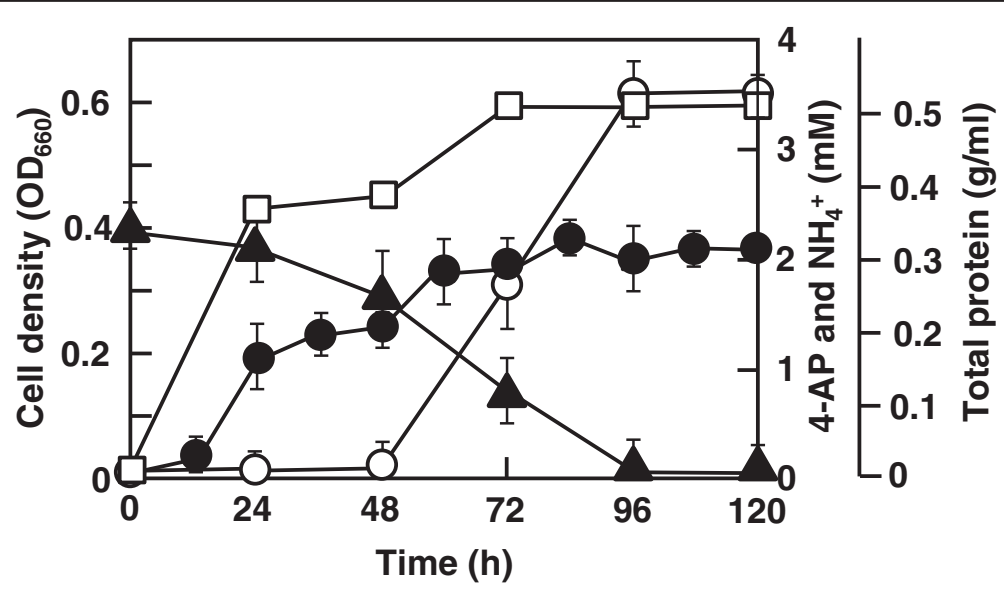

Figure 2 Growth of the enrichment culture in medium containing 4-aminopyridine. Growth and degradation of 4-aminopyridine. The enrichment culture was cultivated in medium containing $2.13 \mathrm{mM} 4$-aminopyridine $(0.02 \% \mathrm{wt} / \mathrm{vol})$ at $30^{\circ} \mathrm{C}$ with shaking. Growth was determined by measuring the optical density at $660 \mathrm{~nm}\left(\mathrm{OD}_{660}\right)$ (open squares); the residual 4-aminopyridine (filled triangles, 4-AP) was measured using HPLC as described in the text; the released ammonia (open circles) was measured using the indophenol method [21]; and total protein in the culture (filled circles) was measured using the modified Lowry method, independently performed twice. 


\section{Isolation of culturable bacterial strains from the enrichment culture}

The enrichment culture contained at least seven strains of dominant bacteria (designated as strains 4AP-A to 4AP-G) that could grow on nutrient agar. The physiological and biochemical parameters of strains 4AP-A and 4AP-G were characterized, and their 16S rRNA genes were analyzed by sequencing. Strains 4AP-B, 4AP-C, 4AP-D, 4AP-E, and 4AP-F were classified by $16 \mathrm{~S}$ rRNA gene analysis (Table 2, see Additional file 1: Tables S1 and S2). None of these strains could degrade 4aminopyridine by itself or when combined with other strains, including all six of the other culturable dominant strains.

When ten-fold-diluted enrichment culture was spread on agar plates containing 4-aminopyridine, several small colonies appeared. Colony PCR analysis of the $16 \mathrm{~S}$ rRNA gene indicated that these were colonies of strains 4AP-A, identified as a species of Pseudomonas and 4AP-G, identified as a species of Enterobacter. Attempts to isolate 4-aminopyridine-degrading bacteria by changing the concentration of 4-aminopyridine and the incubation period at $30^{\circ} \mathrm{C}$ were unsuccessful. We could, however, isolate large colonies of strain 4AP-A on an agar plate containing 3,4-dihydroxypyridine.

\section{DGGE analysis of the enrichment culture}

The enrichment culture grown in $2.13 \mathrm{mM}$ 4aminopyridine medium was used to inoculate fresh medium containing 4-aminopyridine, and aliquots of the new, growing culture were collected in the early-, mid-, and late-exponential growth phases as described in the Materials and methods section. In DGGE gels, the intensity of the bands of some samples increased with the degradation of 4-aminopyridine, and two main bands were present at the same intensity in all samples throughout growth (Figure 3). These two main bands were assigned to strains 4AP-A and 4AP-G based on sequence analysis of the V3 regions of the $16 \mathrm{~S}$ rRNA gene from those two main bands and of the complete $16 \mathrm{~S}$ rRNA gene from culturable strains 4AP-A to 4AP-G.

We then cultivated the enrichment culture in medium containing various concentrations of 4-aminopyridine to reveal the effect of the compound on the abundance of the dominant bacteria. The intensity of a new band (assigned to strain 4AP-Y) increased with the 4aminopyridine concentration (Figure 4), whereas the intensity of the bands assigned to strains 4AP-A and 4AP-G decreased.

To clarify the role of strain 4AP-Y in the biodegradation of 4-aminopyridine, we diluted the enrichment culture $10^{8}$-fold in $0.8 \%$ (wt/vol) $\mathrm{NaCl}$ solution and used it to inoculate 40 tubes of medium containing $2.13 \mathrm{mM} 4$ aminopyridine, yeast extract, and soil extract. The optical density at $660 \mathrm{~nm}$ gradually and similarly increased in all subcultures. However, the rates of 4-aminopyridine degradation in the 40 subcultures differed. We compared the bacteria in the three subcultures that completely degraded 4-aminopyridine in 4 days (Figure 5A, subcultures a, b, and c) with the subculture that did not degrade the substrate (Figure 5A, subculture d). DGGE analysis showed that those subcultures that degraded 4aminopyridine contained strain $4 \mathrm{AP}-\mathrm{Y}$ as a predominant strain (Figure 5B, subcultures a, b, and c), whereas the subculture that did not degrade 4-aminopyridine did not contain strain 4AP-Y (Figure 5B, subculture d).

The full-length sequence of the $16 \mathrm{~S}$ rRNA gene of strain 4AP-Y showed a high level of identity with that of a Hyphomicrobium species detected in a wastetreatment plant (AF098790, [24]) and of unculturable Hyphomicrobium species detected by PCR-DGGE (FJ889298, 4; FJ536932, [25]) (Additional file 1: Table S2). Species of the genus Hyphomicrobium form characteristic mother cells with hyphae and can utilize C1 compounds, e.g., methanol, formate, or methylamine [26]. We observed bi-polar filamentous cells with this shape in the culture grown with 4-aminopyridine (see Additional file 2: Figure S2). Our attempts to isolate Hyphomicrobium sp. strain 4AP-Y using medium containing methanol, formate,

Table 2 Identification of bacteria constituting the 4-aminopyridine-degrading enrichment culture

\begin{tabular}{llll}
\hline Strain & Genus or species affiliation (RDP II classifier) & Best database match & Identity (\%) \\
\hline 4AP-A & Pseudomonas nitroreducens & P. nitroreducens IAM 1439 (AM088473) & 1511/1523 (99.1\%) \\
\hline 4AP-B & Stenotrophomonas maltophilia & S. maltophilia e-p13 (AJ293473) & $1532 / 1537(99.7 \%)$ \\
\hline 4AP-C & Enterobacter agglomerans & E. agglomerans JCM1236 (AB004691) & $1514 / 1536(99.3 \%)$ \\
\hline 4AP-D & Tsukamurella pulmonis & T. pulmonis NIPHL170804 (AY741505) & $1505 / 1515(99.1 \%)$ \\
\hline 4AP-E & Burkholderia & B. cenocepacia J2315 (AM747721) & $1523 / 1525(99 \%)$ \\
\hline 4AP-F & Microbacterium & M. esteraromaticum S29 (AB099658) & $1509 / 1519(99 \%)$ \\
\hline 4AP-G & Enterobacter & Enterobacter sp. SPh (FJ405367) & $1494 / 1501$ (99\%) \\
\hline 4AP-Y & Hyphomicrobium & Uncultured Hyphomicrobium sp. (FJ889298) & $1427 / 1437$ (99\%) \\
\hline 4AP-Z & Elizabethkingia & E. meningoseptica R3-4A (HQ154560) & $1043 / 1046(99.7 \%)$ \\
\hline
\end{tabular}




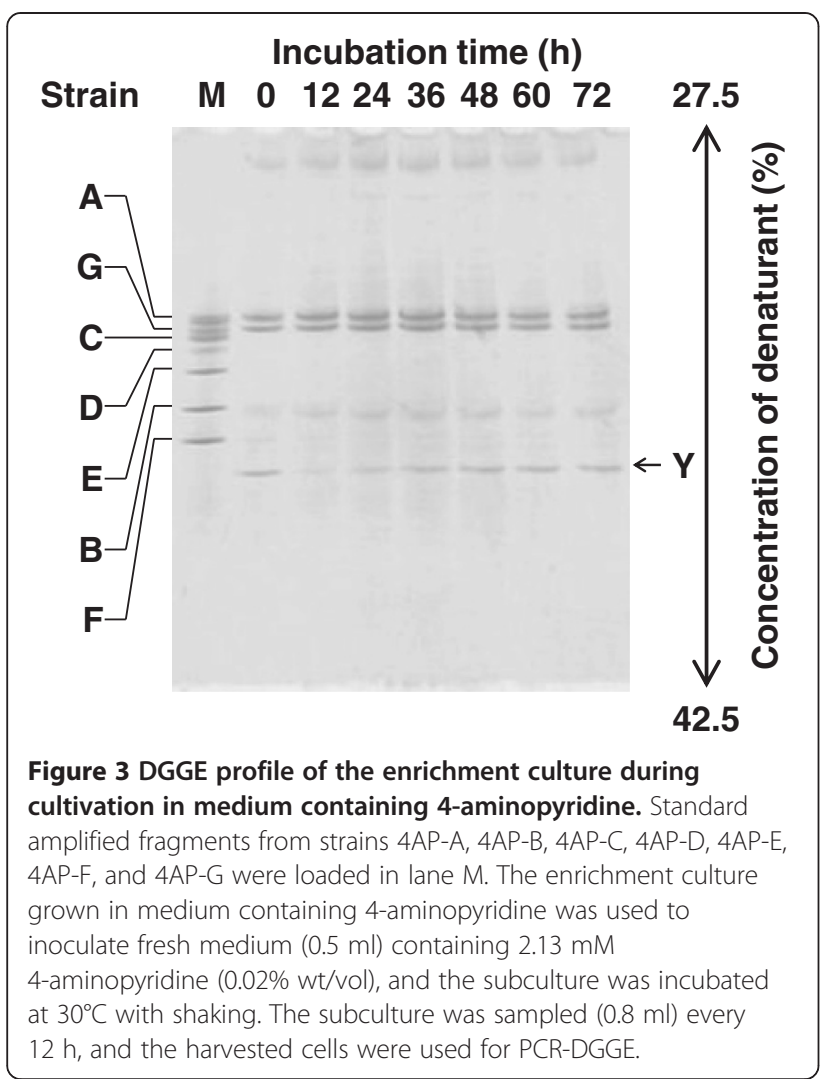

or formamide according to previously reported methods [26] failed.

We analyzed the bacteria in a culture grown with 3,4dihydroxypyridine by PCR-DGGE (Figure 6A). The culture completely degraded 3,4-dihydroxypyridine during 4 days of cultivation. Among the dominant bacteria, strain 4AP-A grew well in the 3,4-dihydroxypyridine medium and completely degraded 3,4-dihydroxypyridine during 3 days of cultivation. Strain 4AP-G grew slowly and degraded the substrate in 7 days. In the DGGE gels, several bands, including that of strain 4AP-A, were present; the band corresponding to strain 4AP-Y was absent; and a new band appeared. The sequence of the $16 \mathrm{~S}$ rRNA gene of the bacterium corresponding to the new band, strain 4AP-Z, showed a high level of identity with those of Elizabethkingia spp. (GU084120 and AY468482). We also analyzed the bacteria in a culture grown with formate by PCR-DGGE (Figure 6B). In the DGGE gels, several bands, including that of strain Y, were present.

\section{Discussion}

The pyridine-ring hydroxylation step is one of main initial steps in the degradation of pyridines [4]. Our analyses of the accumulated metabolites from 4aminopyridine and the growth substrate specificity suggested that 4-aminopyridine was converted to 4-

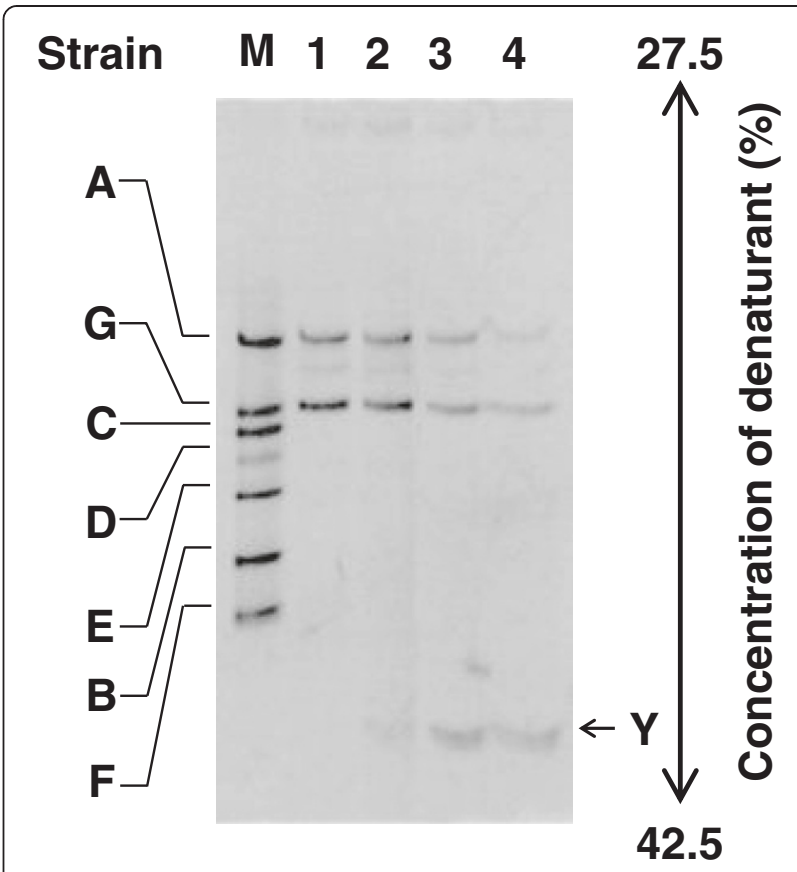

Figure 4 DGGE profile of the enrichment culture grown in media containing various concentrations of 4-aminopyridine. The enrichment culture was used to inoculate basal medium without 4-aminopyridine (lane 1) and with 4-aminopyridine (lane 2, $2.13 \mathrm{mM}$; lane 3, $10.6 \mathrm{mM}$; and lane 4, $53.2 \mathrm{mM}$ ), and the subcultures were incubated at $30^{\circ} \mathrm{C}$ with shaking. After 4 days of cultivation, the subcultures were sampled for PCR-DGGE analysis. The standard amplified fragments from strains $4 A P-A, 4 A P-B, 4 A P-C, 4 A P-D, 4 A P-E$, $4 \mathrm{AP}-\mathrm{F}$, and $4 \mathrm{AP}-\mathrm{G}$ were loaded in lane $\mathrm{M}$.

amino-3-hydroxypyridine and 3,4-dihydroxypyridine (Figure 1). We hypothesized that 4-hydroxypyridine is another possible metabolite based on the previously reported metabolic pathways of pyridines [3]. The enrichment culture could not degrade 4-amino-3-hydroxypyridine and 4hydroxypyridine, even when 4-aminopyridine was added to the medium. Therefore, 4-amino-3-hydroxypyridine must be a dead-end product. In the enrichment culture, 4aminopyridine probably would be directly converted to 3,4-dihydroxypyridine mainly by dehydroxylation and the release of ammonia (Figure 1), similar to the conversion of aniline to benzenediol (catechol) by a dioxygenase [27].

How 3,4-dihydroxypyridine is further metabolized in the enrichment culture is not known, but in Agrobacterium sp. NCIB 10413, 3,4-dihydroxypyridine is converted to 3 -formiminopyruvate via the putative intermediate $3-(\mathrm{N}$ formyl)-formiminopyruvate by the $\mathrm{N}$-heterocyclic ringcleavage dioxygenase, 3-hydroxy-4-pyridone dioxygenase (3,4-dihydroxypyridine 2,3-dioxygenase) [6,7]. The gene encoding 3-hydroxy-4-pyridone dioxygenase, $p y d A$, from Rhizobium sp. TAL1145 has been cloned, and the pyd gene cluster (AY729020) involved in the degradation and transport of 3-hydroxy-4-pyridone has been functionally analyzed [28]. However, the dioxygenases from strains 


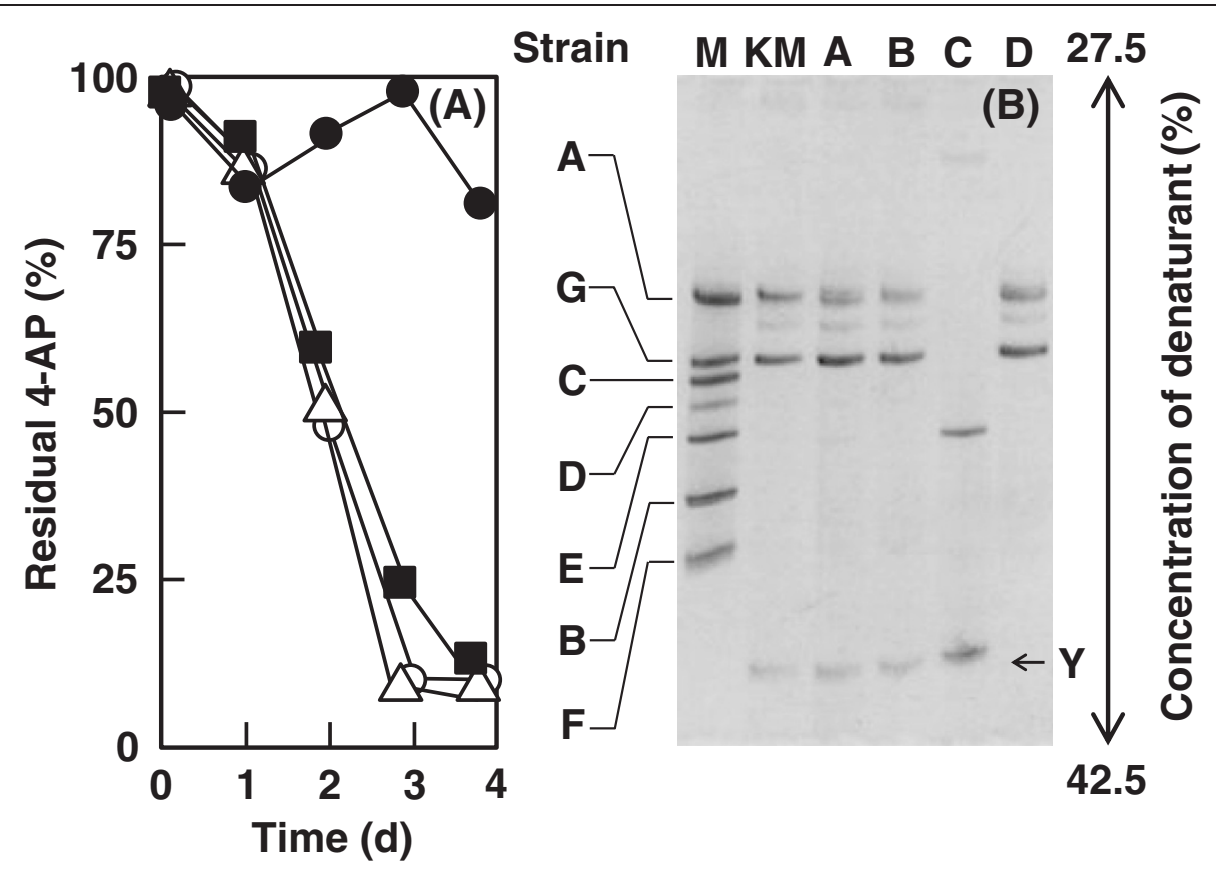

Figure 5 DGGE profile of the enrichment cultures from a diluted pre-culture sample. (A) Degradation of 4-aminopyridine by the diluted enrichment culture. The enrichment culture grown in medium containing 4-aminopyridine was diluted $10^{8}$-fold with $0.8 \% \mathrm{NaCl}$ solution, and the diluted culture was used to inoculate fresh medium containing $2.13 \mathrm{mM} \mathrm{4-aminopyridine;} \mathrm{the} \mathrm{subculture} \mathrm{was} \mathrm{incubated} \mathrm{at} 30^{\circ} \mathrm{C}$ with shaking. The remaining 4-aminopyridine (4-AP) was measured using HPLC as described in the text. (Subcultures: a, open triangles; b, open circles; and c, filled squares; $d$, filled circles). The results of one representative experiment are shown; the residual 4-aminopyridine was measured in triplicate. (B) DGGE profiles of the enrichment culture. Subcultures that degraded 4-aminopyridine in 4 days $(a, b$, and $c)$ and the subculture that did not degrade 4-aminopyridine (d) were analyzed by PCR-DGGE. The standard amplified fragments from strains 4AP-A, 4AP-B, 4AP-C, 4AP-D, 4AP-E, $4 A P-F$, and $4 A P-G$ were loaded in lane M. The harvested cells of the enrichment culture were also used for PCR-DGGE (lane KM).

NCIB 10413 and TAL1145 have not yet been purified and characterized. This enzyme is unstable and easily loses activity during cell extract preparation [6,7]. PydA from strain TAL1145 shows a high level of sequence identity with previously reported class III type meta-cleavage dioxygenases including putative 3-hydroxy-4-pyridone dioxygenase (YP_004673996) from Hyphomicrobium sp. $\mathrm{MC1}$. Here, we did not detect dioxygenase activity in the mixed cells harvested from the enrichment culture. In a preliminary study, the partial $p y d A$ gene fragment could be amplified from the cells by using $p y d A$-specific primers. In future studies, we plan on sequencing the entire gene and analyzing its expression with northern blots instead of detecting dioxygenase activity, to obtain support for our proposed metabolic pathway for 4-aminopyridine.

DGGE analyses indicated that Hyphomicrobium sp. strain 4AP-Y is a prominent degrader of 4-aminopyridine in the enrichment culture (Figures 3, 4, and 5) and that strain 4AP-Y is outnumbered in 3,4-dihydroxypyridine medium (Figure 6A). Therefore, strain 4AP-Y probably converts 4-aminopyridine to 3,4-dihydroxypyridine (Figure 1). 3,4-Dihydroxypyridine, which is also formed from L-mimosine by intestinal bacteria, can be degraded by a much wider range of soil bacteria and ruminal bacteria than has been recognized previously [23,29,30]. 3,4-Dihydroxypyridine might be more easily degraded than 4-aminopyridine by the other strains in our enrichment culture, including strains 4AP-A and 4AP-Z (Figure 1).

Hyphomicrobium spp. closely related to strain 4AP-Y have been isolated from waste-water plants [24] or detected as unculturable bacteria by PCR-DGGE [25,31]. Species of the genus Hyphomicrobium are oligocarbophilic and can grow on mineral salt medium, and the growth can be stimulated by soil extract [26]. In addition, they grow well on $\mathrm{C} 1$ compounds, such as methanol, methylated amines or formate [26]. However, little is known about the assimilation of aromatic compounds by Hyphomicrobium spp. [32]. The unculturable Hyphomicrobium sp. Y17-2 becomes numerically dominant in enrichment cultures containing toluene and $o$-xylene [33]. In our enrichment culture, Hyphomicrobium sp. 4AP-Y probably plays an important role in the initial step of 4-aminopyridine degradation. Other dominant strains, such as strains 4AP-A and 4AP-Z probably share 3,4-dihydroxypyridine and its metabolites as growth substrates. Strain 4AP-Y probably utilizes one of final metabolites from 3,4-dihydroxypyridine, i.e., formate, via the further degradation of this intermediate by other dominant strains. 


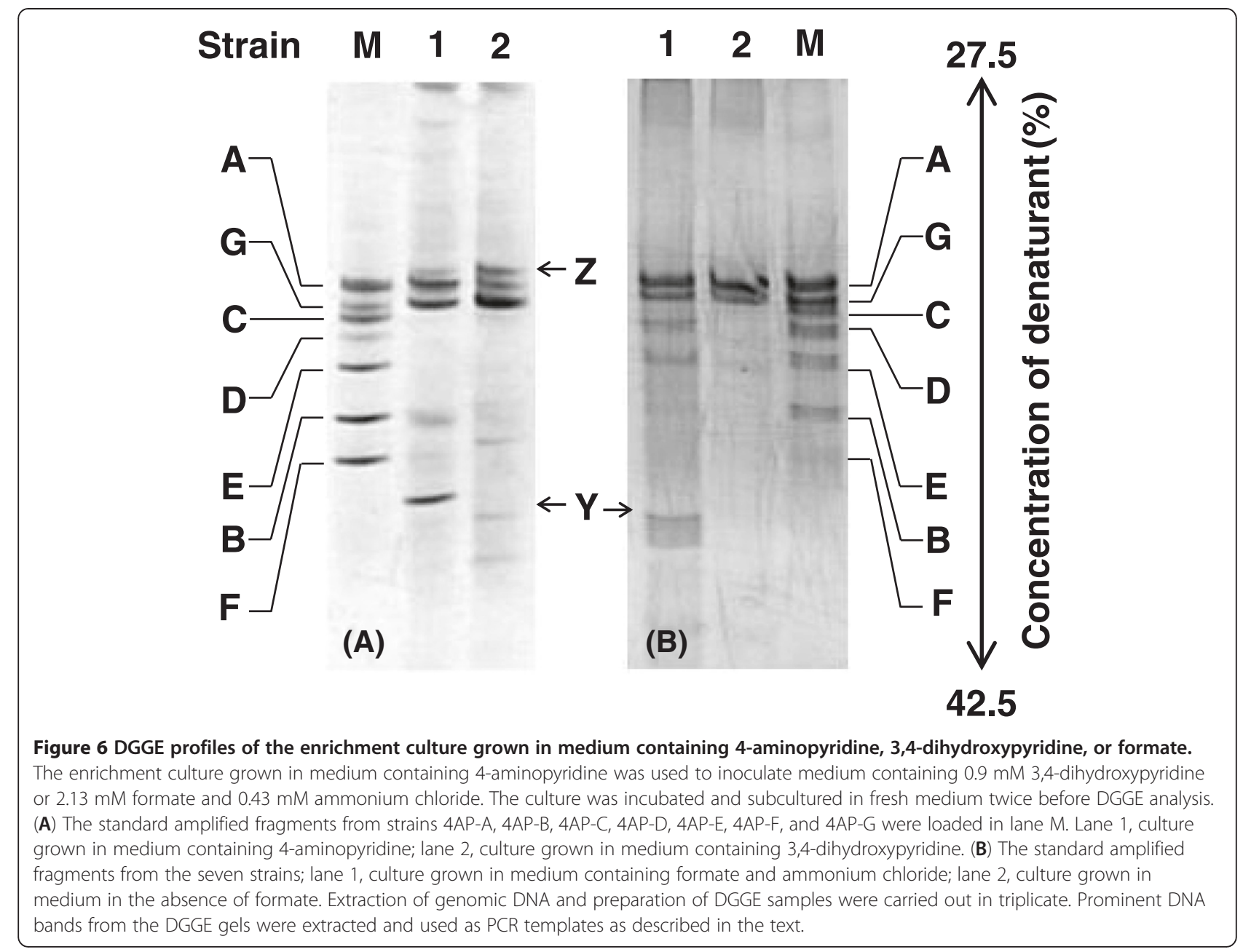

The phytotoxicity, absorption, and translocation of 4aminopyridine in corn and sorghum growing in treated nutrient cultures and soils have been examined by Starr and Cunningham [34]. Although aerobic and anaerobic degradation of 4-aminopyridine in soil had been expected, the authors found little evidence to support biodegradation. Our data reported here indicated that 4aminopyridine can be mineralized by soil microbiota, and we identified bacteria possibly involved in the degradation. To further elucidate the degradation, we will need to establish culture conditions for the isolation of strain 4AP-Y to be able to study the enzymes involved in the degradation of 4-aminopyridine.

\section{Conclusions}

We isolated a 4-aminopyridine-degrading enrichment culture from a normal soil sample, revealed the metabolic fate of 4-aminopyridine, and characterized the bacterial population in the culture. GC-MS analysis and growth substrate specificity indicated that 4-aminopyridine was probably metabolized to 3,4-dihydroxypyridine and that formate probably is one of metabolites. DGGE analysis revealed that the unculturable strain, Hyphomicrobium sp. strain 4AP-Y became more dominant with increasing 4aminopyridine concentration in the culture and in the presence of formate and Elizabethkingia sp. 4AP-Z was dominant in the presence of 3,4-dihydroxypyridine. Hyphomicrobium sp. strain 4AP-Y, Elizabethkingia sp. 4AP-Z, and the culturable 3,4-dihydroxypyridine-degrading bacterium, Pseudomonas nitroreducens 4AP-A and Enterobacter sp. 4AP-G probably play important roles in 4-aminopyridine degradation.

\section{Additional files}

Additional file 1: Table S1. Identification of strains in the 4aminopyridine-degrading enrichment culture. Table S2. 165 rRNA gene analysis of the predominant bacteria in the 4-aminopyridine-degrading enrichment culture.

Additional file 2: Figure S1. Alignment of the partial sequence of the putative 3-hydroxy-4-pyridone dioxygenase (PydA) from 3, 4-dihydroxypyridine-degrading bacteria with sequences of previously 
reported PydAs. Figure S2. Micrograph of cells of the enrichment culture growing in medium containing 4-aminopyridine.

\section{Competing interests}

The authors declare that they have no competing interests.

\section{Authors' contributions}

All authors contributed in the organization and design of experiments as well as data interpretation and manuscript preparation. RN and AM isolated the 4-aminopyridine-degrading enrichment culture and identified the culturable bacteria. RN performed the DGGE analysis. ST separated and identified the metabolites. ST and KY wrote the manuscript. All authors read and approved the final version of the manuscript.

\section{Acknowledgements}

We would like to thank Prof. Hirosato Takiwaka for helping with the chemical synthesis of 3,4-dihydroxypyridine and NMR analysis.

Received: 4 January 2013 Accepted: 11 March 2013

Published: 21 March 2013

\section{References}

1. Hollins RA, Merwin LH, Nissan RA, Wilson WS, Gilardi R: Aminonitropyridines and their N-oxides. J Heterocycl Chem 1996 33(3):895-904

2. Liu S-M, Wu C-H, Hung H-J: Toxicity and anaerobic biodegradability of pyridine and its derivatives under sulfidogenic conditions. Chemosphere 1998, 36(10):2345-2357.

3. Kaiser JP, Feng Y, Bollag JM: Microbial metabolism of pyridine, quinoline, acridine, and their derivatives under aerobic and anaerobic conditions. Microbiol Rev 1996, 60(3):483-498

4. Fetzner S: Bacterial degradation of pyridine, indole, quinolone, and their derivatives under different redox conditions. Appl Microbiol Biotechnol 1998, 49(3):237-250

5. Lee JJ, Rhee S-K Lee S-T: Degradation of 3-methylpyridine and 3-ethylpyridine by Gordonia nitida LE31. Appl Environ Microbiol 2001, 67(9):4342-4345.

6. Watson GK, Houghton C, Cain RB: The hydroxylation of 4-hydroxypyridine to pyridine-3,4-diol (3,4-dihydroxypyridine) by 4-hydroxypyridine-3hydroxylase. Biochem J 1974, 140(2):265-276.

7. Watson GK, Houghton C, Cain RB: Microbial metabolism of the pyridine ring. The metabolism of pyridine-3,4-diol (3,4-dihydroxypyridine) by Agrobacterium sp. Biochem J 1974, 140(2):277-292

8. Zefirov NS, Agapova SR, Terentiev PB, Bulakhova IM, Vasyukova NI, Modyano LV: Degradation of pyridine by Arthrobacter crystallopoietes and Rhodococcus opacus strains. FEMS Microbiol Lett 1994, 118(1-2):71-74.

9. Bai Y, Sun $Q$, Zhao C, Wen D, Tang X: Simultaneous biodegradation of pyridine and quinoline by two mixed bacterial strains. Appl Microbial Biotechnol 2009, 82(5):963-973.

10. Lodlha B, Bhadane R, Patel B, Killedar D: Biodegradation of pyridine by an isolated bacterial consortium/strain and bio-augmentation of strain into activated sludge to enhance pyridine biodegradation. Biodegradation 2008, 19(5):717-723.

11. Vanhoenacker G, Dumont E, David F, Baker A, Sandra P: Determination of arylamines and aminopyridines in pharmaceutical products using in-situ derivatization and liquid chromatography-mass spectrometry. J Chromatog A 2009, 1216(16):3563-3570.

12. Stickley AR, Mitchell RT, Health RG, Ingram CR, Bradly EL: A method for appraising the bird repellency of 4-aminopyridine. J Wildlife Manage 1972, 36(4):1313-1316

13. Ogita K, Okuda H, Watanabe M, Nagashima R, Sugiyama C, Yoneda Y: In vivo treatment with the $\mathrm{K}^{+}$channel blocker 4-aminopyridine protects against kainate-induced neuronal cell death through activation of NMDA receptors in murine hippocampus. Neuropharmacology 2005, 48(6):810-821.

14. Yamaguchi S, Rogawski MA: Effects of anticonvulsant drugs on 4-aminopyridine-induced seizures in mice. Epilepsy Res 1992, 11(1):9-16.

15. Fragoso-Veloz J, Massieu L, Alvarado R, Tapia R: Seizures and wet-dog shake induced by 4 -aminopyridine, and their potentiation by nifedipine. Euro J Pharmacol 1990, 178(3):275-284.

16. Betts PM, Giddings CW, Fleeker JR: Degradation of 4-aminopyridine in soil. J Agric Food Chem 1976, 24(3):571-574.
17. Takenaka S, Asami T, Orii C, Murakami S, Aoki K: A novel meta-cleavage dioxygenase that cleaves a carboxyl-group-substituted 2-aminophenol. Eur J Biochem 2002, 269(23):5871-5877.

18. Edward U, Rogall T, Blöcker H, Emde M, Böttger EC: Isolation and direct complete nucleotide determination of entire genes. Characterization of a gene coding for 165 ribosomal RNA. Nuc Acids Res 1989, 17(19):7843-7853.

19. Kage $\mathrm{S}$, Kudo $\mathrm{K}$, Ikeda $\mathrm{H}$, Ikeda $\mathrm{N}$ : Simultaneous determination of formate and acetate in whole blood and urine from humans using gas chromatography-mass spectrometry. J Chromatogr B 2004, 805(1):113-117.

20. Ovreås L, Forney L, Daae FL, Torsvik V: Distribution of bacterioplankton in meromictic Lake Saelenvannet, as determined by denaturing gradient gel electrophoresis of PCR-amplified gene fragments coding for $16 \mathrm{~S}$ rRNA. Appl Environ Microbiol 1997, 63(9):3367-73.

21. Weatherburn MW: Phenol-hypochlorite reaction for determination of ammonia. Anal Chem 1967, 39(8):971-4.

22. Hartree EF: Determination of protein: a modification of the Lowry method that gives a linear photometric response. Anal Biochem 1985, 48(2):422-427.

23. Allison MJ, Hammond AC, Jones RJ: Detection of ruminal bacteria that degrade toxic dihydroxypyridine compounds produced from mimosine. Appl Environ Microbiol 1990, 56(3):590-594

24. Layton AC, Karanth PN, Lajoie CA, Meyers AJ, Gregory IR, Stapleton RD, Taylor DE, Sayler GS: Quantification of Hyphomicrobium populations in activated sludge from an industrial wastewater treatment system as determined by $16 \mathrm{~S}$ rRNA analysis. Appl Environ Microbiol 2000, 66(3):1167-1174

25. Hayes AC, Zhang $Y$, Liss SN, Allen DG: Linking performance to microbiology in biofilters treating dimethyl sulphide in the presence and absence of methanol. App/ Microbiol Biotechnol 2010, 85(4):1151-1166.

26. Gliesche C, Fesefeldt A, Hirsch P: Genus I. Hyphomicrobium Stutzer and Hartleb 1898, $76^{\mathrm{AL}}$. In Bergey's manual of systematic bacteriology. The proteobacteria, part C, The alpha-, beta-, delta-, and epsilonproteobacteria Volume 2. 2nd edition. Edited by Brenner DJ, Krieg NR Staley JT. New York: Springer; 1993:476-494.

27. Murakami S, Hayashi T, Maeda T, Takenaka S, Aoki K: Cloning and functional analysis of aniline dioxygenase gene cluster, from Frateuria species ANA-18, that metabolizes aniline via an ortho-cleavage pathway of catechol. Biosci Biotech Biochem 2003, 67(11):2351-2358.

28. Awaya JD, Fox PM Borthakur D: pyd genes of Rhizobium sp. strain TAL1145 are required for degradation of 3-hydroxy-4-pyridone, an aromatic intermediate in mimosine metabolism. J Bacteriol 2005, 187(13):4480-4487.

29. Dominguez-Bello GM, Stewart CS: Degradation of mimosine, 2,3dihydroxy pyridine and 3-hydroxy-4(1H)-pyridine by bacteria from the rumen of sheep in Venezuela. FEMS Lett 1990, 73(4):283-289.

30. Hammond AC: Leucaena toxicosis and its control in ruminants. J Anim SC 1995, 73(5):1487-1492

31. Ceja-Navarro JA, Rivera-Orduna FN, Patino-Zuniga L, Vila-Sanjurjo A, Crossa J, Govaerts B, Dendooven L: Phylogenetic and multivariate analyses to determine the effects of different tillage and residue management practices on soil bacterial communities. Appl Environ Microbiol 2010, 76(11):3685-3691.

32. Ralebitso TK, Yamazoe A, Reuling WF, Braster M, Senior E, van Verseveld HW: Insights into bacterial associations catabolizing atrazine by culturedependent and molecular approaches. World J Microbiol Biotechnol 2003 19(1):59-67.

33. Ralebitso TK, Roling WFM, Braster M, van Senior E, Verseveld HW: 16S rDNAbased characterization of BTX-catabolizing microbial associations isolated from a South African sandy soil. Biodegradation 2000 11(6):351-357.

34. Starr Rl, Cunningham DJ: Phytotoxicity, absorption, and translocation of 4-aminopyridine in corn and sorghum growing in treated nutrient cultures and soils. J Agric Food Chem 1974, 22(3):409-413.

doi:10.1186/1471-2180-13-62

Cite this article as: Takenaka et al:: Enrichment and characterization of a bacterial culture that can degrade 4-aminopyridine. BMC Microbiology 2013 13:62. 